\title{
Immunohistochemical expression of vascular endothelial growth factor in canine oral squamous cell carcinomas
}

\author{
MANUELA MARTANO ${ }^{1}$, BRUNELLA RESTUCCI ${ }^{1}$, DORA MARIA CECCARELLI ${ }^{1}$, \\ LORENZO LO MUZIO $^{2}$ and PAOLA MAIOLINO ${ }^{1}$ \\ ${ }^{1}$ Department of Veterinary Medicine and Animal Production, University of Naples Federico II, Naples 80137; \\ ${ }^{2}$ Department of Clinical and Experimental Medicine, University of Foggia, Foggia 71122, Italy
}

Received November 9, 2014; Accepted September 14, 2015

DOI: $10.3892 / \mathrm{ol} .2015 .3847$

\begin{abstract}
Angiogenesis is crucial for the growth and metastasis of malignant tumours, and various proangiogenic factors promote this process. One of these factors is vascular endothelial growth factor (VEGF), which appears to play a key role in tumour angiogenesis. The aim of the present study was to assess whether VEGF expression is associated with angiogenesis, disease progression and neoplastic proliferation in canine oral squamous cell carcinoma (OSCC) tissue. VEGF immunoreactivity was quantified by immunohistochemistry in 30 specimens, including normal oral mucosa and OSCC tissues graded as well, moderately or poorly differentiated. VEGF expression was correlated with tumour cell proliferation, as assessed using the proliferating cell nuclear antigen (PCNA) marker and microvessel density (data already published). The present results revealed that VEGF and PCNA expression increased significantly between normal oral tissue and neoplastic tissue, and between well and moderately/poorly differentiated tumours. In addition, VEGF expression was strongly correlated with PCNA expression and microvessel density. It was concluded that VEGF may promote angiogenesis through a paracrine pathway, stimulating endothelial cell proliferation and, similarly, may induce tumour cell proliferation through an autocrine pathway. The present results suggest that the evaluation of VEGF may be a useful additional criterion for estimating malignancy and growth potential in canine OSCCs.
\end{abstract}

\section{Introduction}

Oral carcinogenesis is considered to be a sequential and multi-step process that is mediated by the deregulation of

Correspondence to: Dr Manuela Martano, Department of Veterinary Medicine and Animal Production, University of Naples Federico II, 1 Via Delpino, Naples 80137, Italy

E-mail:manuela.martano@unina.it

Key words: vascular endothelial growth factor, dog, oral cancer, oral squamous cell carcinoma, immunohistochemistry crucial molecular pathways, among which angiogenesis has long been considered one of the most important mechanisms involved in the biology of cancer $(1,2)$. Numerous tumours promote growth and dispersion to form metastases by recruiting host blood vessels to grow into the vicinity of the tumour (3-5). Angiogenic switch is determined by the net balance of natural inhibitors and angiogenic stimulators, out of which vascular endothelial growth factor (VEGF), also termed VEGF-A, is considered to be the major stimulator responsible for tumour angiogenesis (6,7). Although extensive data are available on the role of VEGF and VEGF receptors in the biology and development of a variety of human and animal invasive malignant tumours (8-14), little information exists concerning oral canine tumours (15).

The present study hypothesises, however, that an improved understanding of the underlying mechanism of oral cancer pathogenesis in dogs may be essential to improve the diagnosis of canine oral cancer. This may also aid the understanding of the biological behaviour of tumours and the selection of the treatment of patients (16).

These considerations prompted the investigation of VEGF expression using immunohistochemistry in a series of various pathological lesions of oral canine mucosa, raging between normal epithelium and squamous cell carcinoma, with various grades of differentiation. VEGF expression was also associated with tumour cell proliferation, as assessed using the proliferating cell nuclear antigen (PCNA) marker, and microvessel density (MVD).

\section{Materials and methods}

Tissue samples. In total, 30 canine oral tissue specimens were examined, which consisted of 6 normal oral mucosa and 24 oral squamous cell carcinoma (OSCC) tissues from 7 Manchester terriers, 8 English setters, 8 huskies and 7 mixed breed dogs. Samples were obtained following surgery from the Department of Veterinary Medicine and Animal Production, University of Naples. The specimens were fixed in $10 \%$ neutral buffered formalin, embedded in paraffin wax, stained with haematoxylin and eosin (Carlo Erba Reagents SAS, Val de Reuil, France) and classified using the World Health Organization criteria (17). The OSCC tissues were graded by two independent observers, according to the criteria 
proposed by Pindborg (18), as well-differentiated (grade 1; 7 out of 24 tissues), moderately differentiated (grade 2; 9 out of 24 tissues) or poorly differentiated (grade 3;8 out of 24 tissues). The samples did not include SCC of the tonsil, as this lesion is usually considered separately from other OSCCs, due to the differences in its geographical distribution and biological behaviour $(19,20)$.

Out of the 24 OSCC tissues considered in the present study, 8 lesions were analysed in a previous study (8), in which the microvessel density, indicated by the number of microvessels per $\mathrm{mm}^{2}$ of neoplastic tissue, was calculated (8).

Immunohistochemistry. The procedure used for immunohistochemical analysis was the streptavidin-biotin peroxidase method (LSAB kit; Dako, Glostrup, Denmark), which was the same method used in previous studies (12,21-23). Briefly, histological sections $(5 \mu \mathrm{m})$ were dewaxed in xylene, dehydrated in graded concentrations of ethanol and washed in $0.01 \mathrm{M}$ phosphate-buffered saline (PBS; pH 7.2 \pm 7.4 ). Endogenous peroxidase activity was blocked using $0.3 \%$ hydrogen peroxide (Carlo Erba Reagents SAS) in absolute methanol for $30 \mathrm{~min}$. Prior to incubation with primary antibodies, the tissue sections were heated in a microwave oven for 3 cycles of 5 min (500-750 W) each in EDTA buffer (pH 6.0; SigmaAldrich, Irvine, UK). The sections were then incubated overnight at $4^{\circ} \mathrm{C}$ with a monoclonal mouse anti-vascular endothelial growth factor (diluted 1:100 PBS; clone, JH121; cat no. MS35OPO; Neo Markers, Fremont, CA, USA) (24) and a monoclonal mouse anti-proliferating cell nuclear antigen (diluted 1:500 PBS; clone, PC10; cat no. M0879; Dako), which was used to identify proliferating cells. The immunolabelling procedure included negative control sections incubated with PBS instead of the primary antibody. A mixture of biotinylated anti-mouse, anti-rabbit and anti-goat immunoglobulins (LSAB kit; cat no. K0675, Dako), diluted in PBS, was used as secondary antibody and was applied for $30 \mathrm{~min}$. To reveal the immunolabelling, 3,3'-diaminobenzidine tetrahydrochloride was used as a chromogen and haematoxylin was used as a counterstain.

Scoring of immunoreactivity. The immunoreactivity for VEGF and PCNA was evaluated by two observers that selected 20 maximally immunostained regions for each case at a magnification of $x 400$ (x40 objective; $x 10$ ocular). The number of cells expressing VEGF and PCNA were counted individually. At least 1,000 cells were counted to obtain the percentage of positive cells. Epithelial cells exhibiting distinct cytoplasmic staining for VEGF and a distinct brown nuclear reaction to PCNA labelling were considered to express VEGF and PCNA, respectively, and were classified based on the intensity of staining into weak and strong immunoreactivity groups.

Statistical analysis. The percentage of cells expressing VEGF and PCNA in each group (OSCC grades 1-3) was expressed as the mean \pm standard deviation. Statistical analysis was performed using analysis of variance. The correlation between the VEGF and PCNA values was assessed using a linear correlation test. $\mathrm{P}<0.01$ was used to represent a statistically significant different.

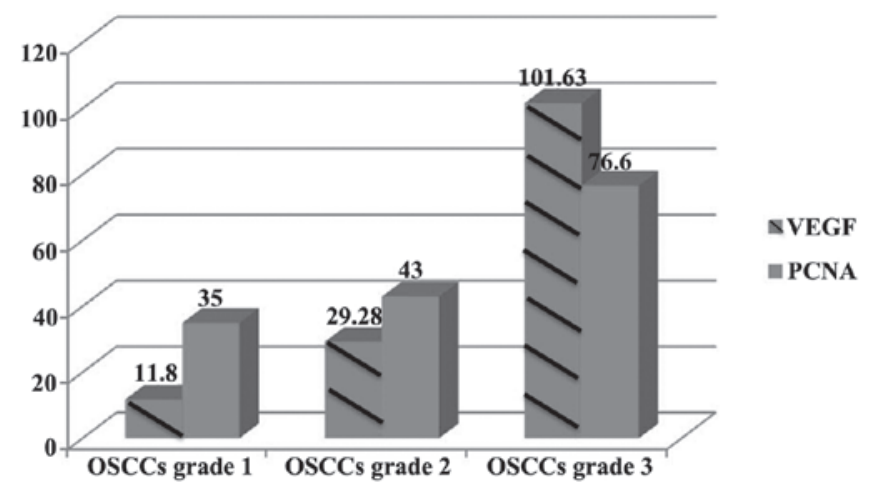

Figure 1. Comparison between the percentage of cells immunolabelled with VEGF and PCNA in samples of canine OSCCs with varying degrees of malignancy (grades 1-3). VEGF, vascular endothelial growth factor; PCNA, proliferating cell nuclear antigen; OSCC, oral squamous cell carcinoma.

\section{Results}

Immunohistochemistry. Results of the immunohistochemical analysis are reported in Table I and Fig. 1.

VEGF expression. VEGF immunopositivity was detected in almost all normal epithelial cells as pale, cytoplasmic staining, which was predominantly detected in the basal layer. Weak VEGF staining was also observed in endothelial cells. In the majority of tumour cells, VEGF expression was indicated by a cytoplasmic staining pattern that ranged between extremely weak and strong, with cytoplasmic granules of variable size and amount. In the majority of grade 1 OSCC tissues (5/7 tissues), VEGF was weakly expressed by epithelial cells, which demonstrated small cytoplasmic granules that were frequently restricted beneath the cytoplasmic membrane (Fig. 2A). In all grade $2(9 / 9)$ and $3(8 / 8)$ OSCC tissues, a heterogeneous pattern of VEGF staining that ranged between moderate and strong in intensity was observed (Fig. 2B). Strong staining was particularly observed in less differentiated regions (Fig. 2C). VEGF granules were large and frequently polarized in grade 2 OSCC tissues and diffuse in the cytoplasm in grade 3 OSCC tissues.

The number of labelled cells increased progressively between grade $1(11.8 \pm 8.86)$ and grade $2(29.28 \pm 20.65)$ OSCC tissues, and an additional increase was observed between grade 2 and grade $3(101.63 \pm 71.96)$ tissues. A significant difference was identified between the number of cells labelled for VEGF expression and the degree of differentiation in OSSC lesions $(\mathrm{P}=0.0008)$.

PCNA expression. PCNA-immunolabelled nuclei were clearly identifiable in normal oral epithelium tissues, as well as in OSCC tissues. In the normal epithelium, weak immunostaining was predominantly observed in the basal layer of the tissue and moderate staining was observed in the parabasal layers, 2-3 layers from the basal layer. In grade 1 and 2 OSCC tissues, moderate nuclear staining was more frequently detected in the peripheral region of the tumour islands and was almost absent in the central keratinized region (Fig. 3A). Grade 3 OSCCs demonstrated strong nuclear immunostaining with a diffuse pattern of staining (Fig. 3B). The number of cells expressing PCNA increased progressively between grade $1(35 \pm 2.23)$ 
Table I. Correlation between the number of cells expressing VEGF and PCNA and the differentiation grades of tumours in canine OSCC tissues.

\begin{tabular}{lccrc}
\hline & \multicolumn{4}{c}{ OSCC grade } \\
\cline { 2 - 4 } Protein expressed & 1 & 2 & 3 & \\
\hline VEGF & $11.80 \pm 8.86$ & $29.28 \pm 20.65$ & $101.63 \pm 71.96$ & 0.0008 \\
PCNA & $35.00 \pm 2.23$ & $43.00 \pm 5.98$ & $76.60 \pm 10.30$ & 0.0010 \\
\hline
\end{tabular}

Data are expressed as the mean number of cells \pm standard deviation. ${ }^{*} \mathrm{P}<0.01$. VEGF, vascular endothelial growth factor; PCNA, proliferating cell nuclear antigen; OSCC, oral squamous cell carcinoma.
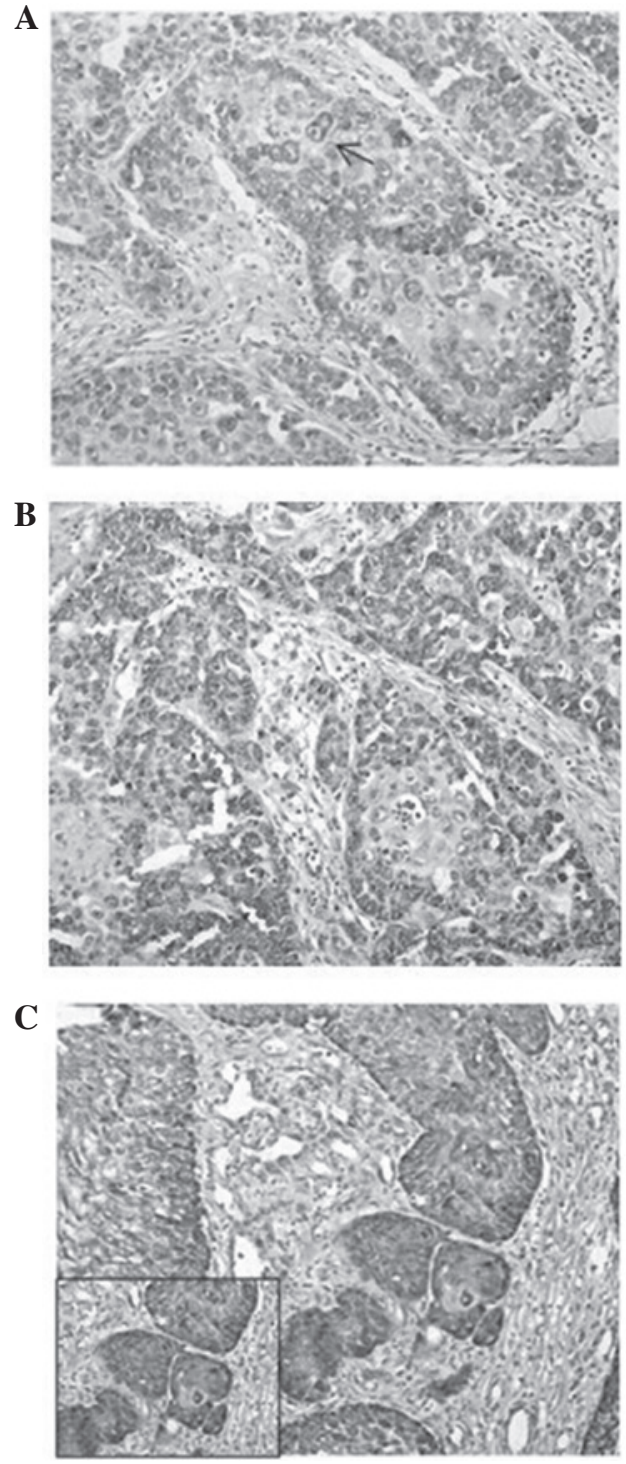

Figure 2. (A) Well-differentiated (grade 1) OSCC tissue demonstrating weak expression of the VEGF protein in few neoplastic cells. The immunolabelling is often restricted often beneath the cytoplasmic membrane (arrow; stain, streptavidin-biotin peroxidase; magnification, x20). (B) Moderately differentiated (grade) OSCC tissue demonstratign moderate expression of the VEGF protein in numerous neoplastic cells. The VEGF expression was localized in almost all the cytoplasm, and was occasionally polarized (stain, streptavidin-biotin peroxidase; magnification, x20). (C) Poorly differentiated (grade 3) OSCC tissue demonstrating strong expression of the VEGF protein in almost all neoplastic cells, with diffuse large granules in the cytoplasm (stain, streptavidin-biotin peroxidase; magnification, $\mathrm{x} 20$; insert magnification, $\mathrm{x} 40$ ). VEGF, vascular endothelial growth factor; OSCC, oral squamous cell carcinoma.
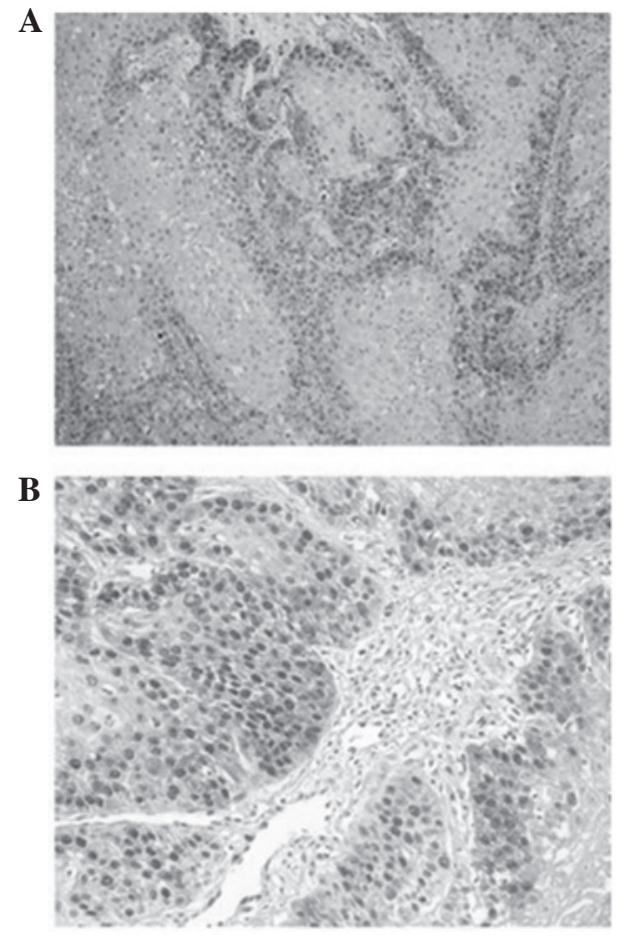

Figure 3. (A) Moderately differentiated (grade 2) OSCC tissue demonstrating moderate intensity nuclear staining for PCNA, detected in the peripheral region of the tumour islands. (stain, streptavidin-biotin peroxidase; magnification, x20). (B) Poorly differentiated (grade 3) OSCC tissue demonstrating strong expression of the PCNA protein by almost all neoplastic cells (stain, streptavidin-biotin peroxidase; magnification, $x 40$ ). VEGF, vascular endothelial growth factor; PCNA, proliferating cell nuclear antigen.

and $2(43 \pm 5.98)$ OSCC tissues and grade 3 OSCC tissues (76.6 \pm 10.30$)$. A significant difference was identified between the number of cells labelled for PCNA expression and the degree of differentiation of the tumours $(\mathrm{P}=0.001)$.

Correlation between the expression of VEGF and PCNA and $M V D$. The expression of VEGF and PCNA strongly correlated with the degree of differentiation of OSCC lesions ( $r=0.999)$. In addition, in 8/24 OSCCs, the MVD (8) strongly correlated with the expression of VEGF $(r=0.993)$ and PCNA $(r=0.993)$.

\section{Discussion}

Squamous cell carcinoma of the oral cavity (OSCC) is the second most common malignant neoplasm of the oral cavity 
in dogs, following melanoma, as it accounts for $17-25 \%$ of canine oral tumours (19). Despite the relatively low metastatic rate $(20 \%)$, OSCC grows rapidly and typically invades nearby bone and tissue, in addition to possessing an extremely high frequency of local recurrence $(18,25)$. In order to improve the understanding of the pathogenesis of this tumour, the present study examined the immunohistochemical expression of VEGF in canine OSCCs and the association of VEGF expression with neoplastic proliferation and microvessel density.

VEGF is a multifunctional cytokine that is produced by neoplastic cells, macrophages, plasma cells and lymphocytes $(26,27)$. This cytokine acts on endothelial cells as a highly specific mitogen, binding to specific class III receptor tyrosine kinases, termed VEGFR-1 (Flt-1) and VEGFR-2 (Flk-1) $(28,29)$. The activation of VEGFR-1 promotes endothelial cell migration, but does not induce cell proliferation (30-32). However, VEGFR-2 is the major mediator of endothelial cell proliferation and survival, and it is also essential for the differentiation of endothelial cells and the induction of microvessel permeability $(11,33)$.

Previous studies investigating the role of VEGF in human oral cancer progression are contradictory. Numerous human cancers have demonstrated increased VEGF expression compared with normal tissue (29,34-36). By contrast, certain studies have reported opposite results, demonstrating that VEGF expression levels were increased in the corresponding normal mucosa epithelium and early stage of premalignant lesions compared with later stages of cancerogenesis $(37,38)$. These previous studies hypothesized that VEGF may perform a physiological role in oral mucosa epithelium, or that the protein may play an important role only in the early stage of oral tumorigenesis, while other genetic factors are involved in the later stages. These discrepancies were considered, and it was suggested that they may be due to the use of different antibodies or quantification methods (39).

In the present study, a significant increase in VEGF expression was identified during the transition between normal and OSCC tissues, in association with an increasing grade of differentiation. This was indicated by a high number of VEGF-positive cells associated with the accumulation and consequent loss of cytoplasmic polarization of VEGF granules in less differentiated tumours. These results are in agreement with those of Sobczyńska-Rak et al (15), who studied the level of VEGF in the serum of dogs with OSCC by ELISA, and identified an elevated presence of VEGF in the blood serum of all examined malignant tumours compared with the benign tumours and control tissues, confirming a significant role of VEGF in the disease progression of canine oral tumours. Upregulation of VEGF, which is particularly found in moderately or poorly differentiated tumours, may be induced mainly by HIF-1 $\alpha$ under the hypoxic conditions present in the necrotic compartments of tumours (40). By contrast, in numerous types of tumours, elevated levels of VEGF production may often be detected in tumour cells located at the extreme periphery of the tumour where there is no hypoxia $(12,41,42)$. Therefore, hypoxia-independent production of VEGF by neoplastic cells may be associated with the activation of oncogenes encoding VEGF, such as the oncogenes that are components of the ras/mitogen-activated protein kinase signal transduction pathway, the inactivation of tumour suppressors, including p53, and exogenous factors, such as hormones and growth factors $(30,43)$.

In addition, in the present study, VEGF expression was found to be significantly correlated with PCNA expression. Tumour growth is associated with the actions of proliferative proteins such as proliferating cell nuclear antigen (PCNA), a non-histamine nuclear protein that is considered a specific marker of cell division $(9,44)$. The expression of PCNA has been found to be correlated with the degree of malignancy and progression of cancer (45). The strong correlation between PCNA and VEGF detected in the present study indicates that VEGF, which is secreted by neoplastic cells, may induce self-proliferation in an autocrine manner, contributing to neoplastic growth, and induce endothelial cell proliferation and tumour angiogenesis through a paracrine mechanism. Therefore, malignant neoplastic cells may act simultaneously as VEGF producers and VEGF target cells, as demonstrated in previous studies $(46,47)$.

Additionally, in the present study, tumour cell proliferation was strongly correlated with microvessel density, demonstrating that increased vascularity occurs to support actively proliferating and transforming oral epithelial cells, in order to permit tumour growth. This finding is in agreement with numerous studies, which have already established that tumour cell proliferation decreases with the increasing distance between the tumour and blood vessels, confirming that tumour growth is strongly dependent on angiogenesis $(9,38,48)$.

Overall, the present results revealed that VEGF levels are elevated in canine OSCC compared with control specimens. Increased expression of this angiogenic factor in less differentiated tumours may determine an increase in neoplastic and blood vessel proliferation, as determined by the strong correlation that was identified between VEGF expression, cell proliferation and microvessel density. This is potentially of significance since neoangiogenesis is necessary for the nutrition of the neoplastic cells that demonstrated an increased proliferative rate, and neoangiogenesis also enhances the ability of tumour cells to migrate, and therefore the potential of the cells to metastasize $(2,32)$. As a result, an increase in VEGF synthesis is associated with increased endothelial and neoplastic cell proliferation, and the risk of tumour growth and metastasis is increased. To confirm this hypothesis, additional studies are required to fully elucidate the role of VEGF and VEGF receptors in tumour-associated angiogenesis and to determine whether receptors and ligands were each expressed in the same cells, indicating an autocrine mechanism, or the receptor was expressed in one compartment and the ligand in one of the other compartments, indicating a paracrine loop (49).

In conclusion, VEGF evaluation may provide additional criteria for assessing the intrinsic malignancy and growth potential of canine oral tumours, and may have a potential application as an indicator of prognosis. In addition, understanding the biology of the VEGF ligand and receptor may facilitate the development of therapeutic strategies to promote revascularization of ischemic tissue or to inhibit angiogenesis in neoplastic pathologies (50). Observations in vivo suggest that the inhibition of VEGF-induced angiogenesis results in suppression of tumour growth in animal studies (51) and a reduction in tumour size and metastasis in vivo (52). 


\section{References}

1. Hasina R and Lingen MW: Angiogenesis in oral cancer. J Dent Educ 65: 1282-1290, 2001.

2. Ascani G, Balercia P, Messi M, Lupi L, Goteri G, Filosa A, Stramazzotti D, Pieramici T and Rubini C: Angiogenesis in oral squamous cell carcinoma. Acta Otorhinolaryngol Ital 25: 13-17, 2005.

3. Blood $\mathrm{CH}$ and Zetter BR: Tumor interactions with the vasculature: Angiogenesis and tumor metastasis. Biochim Biophys Acta 1032: 89-118, 1990 .

4. Folkman J: What is the evidence that tumors are angiogenesis dependent? J Natl Cancer Inst 82: 4-6, 1990.

5. Fox SB, Gatter KC and Harris AL: Tumour angiogenesis. J Pathol 179: 232-237, 1996.

6. Ferrara $\mathrm{N}$ and Keyt B: Vascular endothelial growth factor: Basic biology and clinical implications. EXS 79: 209-232, 1997.

7. Hicklin DJ and Ellis LM: Role of the vascular endothelial growth factor pathway in tumor growth and angiogenesis. J Clin Oncol 23: 1011-1027, 2005.

8. Martano M, Maiolino P, Cataldi M and Restucci B: Evaluation of angiogenesis by morphometric analysis of blood vessels in dysplastic and neoplastic lesions of canine gingiva. Vet Res Commun 28 (Suppl 1): 299-301, 2004.

9. Tipoe GL, Jin Y and White FH: The relationship between vascularity and cell proliferation in human normal and pathological lesions of the oral cheek epithelium. Eur J Cancer B Ora Oncol 32B: 24-31, 1996.

10. Khademi B, Soleimanpour M, Ghaderi A and Mohammadianpanah M: Prognostic and predictive value of serum vascular endothelial growth factor (VEGF) in squamous cell carcinoma of the head and neck. Oral Maxillofac Surg 18: 187-196, 2014.

11. Al-Dissi AN, Haines DM, Singh B and Kidney BA: Immunohistochemical expression of vascular endothelial growth factor and vascular endothelial growth factor receptor associated with tumor cell proliferation in canine cutaneous squamous cell carcinomas and trichoepitheliomas. Vet Pathol 44: 823-830, 2007.

12. Restucci B, Borzacchiello G, Maiolino P, Martano M, Paciello O and Papparella S: Expression of vascular endothelial growth factor receptor Flk-1 in canine mammary tumours. J Comp Pathol 130: 99-104, 2004

13. Restucci B, Maiolino P, Paciello O, Martano M, De Vico G and Papparella S: Evaluation of angiogenesis in canine seminomas by quantitative immunohistochemistry. J Comp Pathol 128 : 252-259, 2003.

14. Maiolino P, De Vico G and Restucci B: Expression of vascular endothelial growth factor in basal cell tumours and in squamous cell carcinomas of canine skin. J Comp Pathol 123: 141-145, 2000.

15. Sobczyńska-Rak A, Polkowska I and Silmanowicz P: Elevated vascular endothelial growth factor (VEGF) levels in the blood serum of dogs with malignant neoplasms of the oral cavity. Acta Vet Hung 62: 362-371, 2014.

16. Martano M, Damiano S, Restucci B, Paciello O, Russo V and Maiolino P: Nuclear morphometry in canine acanthomatous ameloblastomas and squamous cell carcinomas. Eur J Histochem 50: 125-130, 2006.

17. Head KW, Cullen JM, Dubielzig RR, Else RW, Misdorp W, Patnaik AK, Tateyama S and van der Gaag I: Histological classification of tumors of the alimentary system of domestic animals (WHO). Armed Forces Institute of Pathology, Washington, DC 2003.

18. Pindborg JJ (ed): Definition of terms related to oral cancer and precancer. In: Oral cancer and precancer. John Wright \& Sons, Bristol pp2-19, 1980. Todoroff RJ and Brodey RS: Oral and pharyngeal neoplasia in the dog: A retrospective survey of 361 cases. J Am Vet Med Assoc 175: 567-571, 1979.

19. Todoroff RJ and Brodey RS: Oral and pharyngeal neoplasia in the dog: A retrospective survey of 361 cases. J Am Vet Med Assoc 175: 567-571, 1979

20. Mas A, Blackwood L, Cripps P, Murphy S, De Vos J, Dervisis N, Martano M and Polton GA: Canine tonsillar squamous cell carcinoma-a multi-centre retrospective review of 44 clinical cases. J Small Anim Pract 52: 359-364, 2011

21. Restucci B, Maiolino P, Martano M, et al: Expression of beta-catenin, E-cadherin and APC in canine mammary tumors. Anticancer Res 27: 3083-3089, 2007.
22. Restucci B, Martano M, DE Vico G, et al: Expression of E-cadherin, beta-catenin and APC protein in canine colorectal tumours. Anticancer Res 29: 2919-2925, 2009.

23. Martano M, Carella F, Squillacioti C, et al: Metallothionein expression in canine cutaneous apocrine gland tumors. Anticancer Res 32: 747-752, 2012

24. Turley H, Scott PA, Watts VM, et al: Expression of VEGF in routinely fixed material using a new monoclonal antibody VG1. J Pathol 186: 313-318, 1998

25. Head KWER and Dubielzig RR: Tumors of the alimentary tract. In: Tumors in Domestic Animals. Press IS (ed.) DJ Meuten, Ames, pp401-pp481, 2002.

26. Ferrara $\mathrm{N}$ and Davis-Smyth T: The biology of vascular endothelial growth factor. Endocr Rev 18: 4-25, 1997.

27. Dvorak HF, Detmar M, Claffey KP, Nagy JA, van de Water L and Senger DR: Vascular permeability factor/vascular endothelial growth factor: An important mediator of angiogenesis in malignancy and inflammation. Int Arch Allergy Immunol 107: 233-235, 1995

28. Ferrara N, Gerber HP and LeCouter J: The biology of VEGF and its receptors. Nat Med 9: 669-676, 2003.

29. Denhart BC, Guidi AJ, Tognazzi K, Dvorak HF and Brown LF: Vascular permeability factor/vascular endothelial growth factor and its receptors in oral and laryngeal squamous cell carcinoma and dysplasia. Lab Invest 77: 659-664, 1997.

30. Neufeld G, Cohen T, Gengrinovitch S and Poltorak Z: Vascular endothelial growth factor (VEGF) and its receptors. FASEB J 13: 9-22, 1999.

31. Margaritescu C, Pirici D, Simionescu C, Mogoantă L, Raica M, Stîngă A, Ciurea R, Stepan A, Stîngă A and Ribatti D: VEGF and VEGFRs expression in oral squamous cell carcinoma. Rom J Morphol Embryol 50: 527-548, 2009.

32. Johnstone S and Logan RM: The role of vascular endothelial growth factor (VEGF) in oral dysplasia and oral squamous cell carcinoma. Oral Oncol 42: 337-342, 2006.

33. Cross MJ and Claesson-Welsh L: FGF and VEGF function in angiogenesis: Signalling pathways, biological responses and therapeutic inhibition. Trends Pharmacol Sci 22: 201-207, 2001.

34. Maeda T, Matsumura S, Hiranuma H, Jikko A, Furukawa S, Ishida $\mathrm{T}$ and Fuchihata $\mathrm{H}$ : Expression of vascular endothelial growth factor in human oral squamous cell carcinoma: It association with tumour progression and p53 gene status. J Clin Pathol 51: 771-775, 1998.

35. Shintani S, Li C, Ishikawa T, Mihara M, Nakashiro K, et al: Expression of vascular endothelial growth factor A, B, C and $\mathrm{D}$ in oral squamous cell carcinoma. Oral Oncol 40: 13-20, 2004.

36. Shang ZJ and Li JR: Expression of endothelial nitric oxide synthase and vascular endothelial growth factor in oral squamous cell carcinoma: Its correlation with angiogenesis and disease progression. J Oral Pathol Med 34: 134-139, 2005.

37. Salven P, Heikkila P, Anttonen A, Kajanti M and Joensuu H: Vascular endothelial growth factor in squamous cell head and neck carcinoma: Expression and prognostic significance. Mod Pathol 10: 1128-1133, 1997.

38. Tae K, El-Naggar AK, Yoo E, Feng L, Lee JJ, Hong WK, Hittelman WN and Shin DM: Expression of vascular endothelial growth factor and microvessel density in head and neck tumorigenesis. Clin Cancer Res 6: 2821-2828, 2000.

39. Baillie R, Harada K, Carlile J, Macluskey M, Schor SL and Schor AM: Expression of vascular endothelial growth factor in normal and tumour oral tissues assessed with different antibodies. Histochem J 33: 287-294, 2001.

40. Levy NS, Chung S, Furneaux H and Levy AP: Hypoxic stabilization of vascular endothelial growth factor mRNA by the RNA-binding protein HuR. J Biol Chem 273: 6417-6423, 1998.

41. Grugel S, Finkenzeller G, Weindel K, Barleon B and Marmé D: Both v-Ha-Ras and v-Raf stimulate expression of the vascular endothelial growth factor in NIH 3T3 cells. J Biol Chem 270: 25915-25919, 1995

42. Rak J, Filmus J, Finkenzeller G, Grugel S, Marmé D and Kerbel RS: Oncogenes as inducers of tumor angiogenesis. Cancer Metastasis Rev 14: 263-277, 1995.

43. Rak J, Mitsuhashi Y, Bayko L, Filmus J, Shirasawa S, Sasazuki T and Kerbel RS: Mutant ras oncogenes upregulate VEGF/VPF expression: Implications for induction and inhibition of tumor angiogenesis. Cancer Res 55: 4575-4580, 1995

44. Tsai ST and Jin YT: Proliferating cell nuclear antigen (PCNA) expression in oral squamous cell carcinomas. J Oral Pathol Med 24: 313-315, 1995 
45. Kato K, Kawashiri S, Yoshizawa K, et al Expression form of p53 and PCNA at the invasive front in oral squamous cell carcinoma: Correlation with clinicopathological features and prognosis. J Oral Pathol Med 40: 693-698, 2011.

46. Millanta F, Silvestri G, Vaselli C, et al: The role of vascular endothelial growth factor and its receptor Flk-1/KDR in promoting tumour angiogenesis in feline and canine mammary carcinomas: A preliminary study of autocrine and paracrine loops. Res Vet Sci 81: 350-357, 2006.

47. Restucci B, Papparella S, Maiolino P and De Vico G: Expression of vascular endothelial growth factor in canine mammary tumors. Vet Pathol 39: 488-493, 2002.

48. Breward CJW, Byrne HM and Lewis CE: Modelling the interactions between tumour cells and a blood vessel in a microenvironment within a vascular tumour. Eur J Appl Math 12: 529-556, 2001.
49. de Jong JS, van Diest PJ, van der Valk P and Baak JP: Expression of growth factors, growth-inhibiting factors and their receptors in invasive breast cancer. II: Correlations with proliferation and angiogenesis. J Pathol 184: 53-57, 1998.

50. Rosen LS: VEGF-targeted therapy: Therapeutic potential and recent advances. Oncologist 10: 382-391, 2005.

51. Bjorndahl M, Cao R, Eriksson A and Cao Y: Blockage of VEGF-induced angiogenesis by preventing VEGF secretion. Circ Res 94: 1443-1450, 2004.

52. Kim KJ, Li B, Winer J, Armanini M, Gillett N, et al: Inhibition of vascular endothelial growth factor-induced angiogenesis suppresses tumour growth in vivo. Nature 362: 841-844, 1993. 